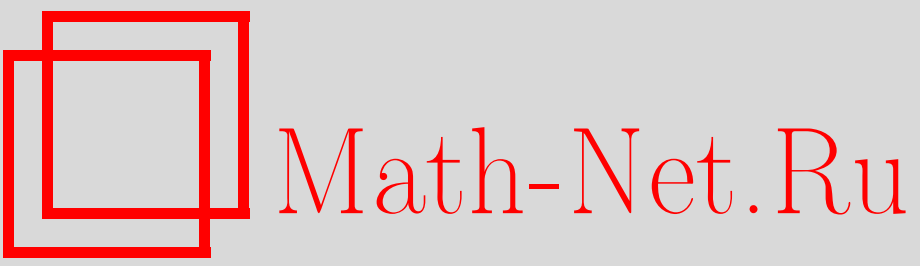

А. В. Ершов, О $K$-теории расслоений на матричные алгебры, УМH, 2000, том 55, выпуск 2, 137-138

DOI: https://doi.org/10.4213/rm276

Использование Общероссийского математического портала Math-Net.Ru подразумевает, что вы прочитали и согласны с пользовательским соглашением

http://www.mathnet.ru/rus/agreement

Параметры загрузки:

IP : 54.198 .64 .247

26 апреля 2023 г., $16: 30: 36$ 


\title{
О К-ТЕОРИИ РАССЛОЕНИЙ НА МАТРИЧНЫЕ АЛГЕБРЫ
}

\author{
А. В. Ершов
}

В работе [1] на категории конечных $C W$-комплексов был определен функтор, принимающий значения в категории абелевых групп и связанный с $K$-теорией специального класса расслоений со слоем матричная алгебра. Там было построено представляющее его пространство. В данной работе доказывается, что оно гомотопически эквивалентно $B S U$ и что соответствующая структура $H$-пространства на $B S U$ изоморфна $H$-структуре, индуцированной тензорным произведением виртуальных расслоений виртуальной размерности 1.

ОПрЕдЕлЕниЕ 1 . Пусть $A_{k}(k \geqslant 1)$ - локально тривиальное векторное расслоение над $X$ со слоем матричная алгебра $M_{k}(\mathbb{C})$ и предположим, что существует отображение расслоений

$$
A_{k} \stackrel{X}{\hookrightarrow}_{{ }^{\mu}}^{\mu} \widetilde{M}_{k l}:=X \times M_{k l}(\mathbb{C})
$$

такое, что для всякой точки $x \in X$ слой $\left(A_{k}\right)_{x} \cong M_{k}(\mathbb{C})$ вкладывается в слой $\left(\widetilde{M}_{k l}\right)_{x} \cong M_{k l}(\mathbb{C})$ как центральная простая подалгебра. Тогда тройку $\left(A_{k}, \mu, \widetilde{M}_{k l}\right)$ назовем расслоением на алгебры (РА) над $X$. Если $k$ и $l$ взаимно просты (наибольший обший делитель $(k, l)=1$ ), то тройку $\left(A_{k}, \mu, \widetilde{M}_{k l}\right)$ назовем плавающим расслоением на алгебры (ПРА).

Отображение $\mathrm{PA}\left(A_{k}, \mu, \widetilde{M}_{k l}\right)$ в $\mathrm{PA}\left(B_{m}, \nu, \widetilde{M}_{m n}\right)$ есть пара отображений расслоений $f: A_{k} \rightarrow B_{m}, g: \widetilde{M}_{k l} \rightarrow \widetilde{M}_{m n}$, являюшихся послойными гомоморфизмами алгебр, такая, что $g \circ \mu=\nu \circ f$.

Тройку $\left(\widetilde{M}_{k}, \tau, \widetilde{M}_{k l}\right)$ назовем тривиальной, если отображение $\tau: X \times M_{k}(\mathbb{C}) \rightarrow X \times M_{k l}(\mathbb{C})$ следующее: $\tau(x, T)=\left(x, T \otimes E_{l}\right)$, где $x \in X, E_{l}$ - единичная $(l \times l)$-матрица и через $T \otimes E_{l}$ обозначено кронекерово произведение матриц.

ОПРЕДЕЛЕНИЕ 2 . Два ПРА $\left(A_{k}, \mu, \widetilde{M}_{k l}\right)$ и $\left(B_{m}, \nu, \widetilde{M}_{m n}\right)$ назовем стабильно әквивалентным.м , если существует последовательность пар натуральных чисел $\left\{t_{i}, u_{i}\right\}, 1 \leqslant i \leqslant s$, такая, что

(i) $\left\{t_{1}, u_{1}\right\}=\{k, l\},\left\{t_{s}, u_{s}\right\}=\{m, n\}$, (ii) $\left(t_{i} t_{i+1}, u_{i} u_{i+1}\right)=1$ при $s>1,1 \leqslant i \leqslant s-1$, и отвечающая ей последовательность ПРА $\left(A_{t_{i}}, \mu_{i}, \widetilde{M}_{t_{i}} u_{i}\right)$ такая, что

(1) $\left(A_{t_{1}}, \mu_{1}, \widetilde{M}_{t_{1} u_{1}}\right)=\left(A_{k}, \mu, \widetilde{M}_{k l}\right),\left(A_{t_{s}}, \mu_{s}, \widetilde{M}_{t_{s}} u_{s}\right)=\left(B_{m}, \nu, \widetilde{M}_{m n}\right)$;

(2) $\left(A_{t_{i}}, \mu_{i}, \widetilde{M}_{t_{i} u_{i}}\right) \otimes\left(\widetilde{M}_{t_{i+1}}, \tau, \widetilde{M}_{t_{i+1} u_{i+1}}\right) \cong\left(A_{t_{i+1}}, \mu_{i+1}, \widetilde{M}_{t_{i+1} u_{i+1}}\right) \otimes\left(\widetilde{M}_{t_{i}}, \tau, \widetilde{M}_{t_{i} u_{i}}\right)$ при $s>1,1 \leqslant i \leqslant s-1$, где $\left(\widetilde{M}_{t_{i}}, \tau, \widetilde{M}_{t_{i} u_{i}}\right)$ - тривиальное ПРА.

Основой РА $\left(A_{k}, \mu, \widetilde{M}_{k l}\right)$ назовем расслоение $A_{k}$, рассматриваемое как расслоение со структурной группой Aut $M_{k}(\mathbb{C})=P G L(k ; \mathbb{C})$.

В [1] были доказаны следуюшие результаты:

А. Структурная группа основы ПРА $\left(A_{k}, \mu, \widetilde{M}_{k l}\right)$ редуцируется к $S U(k)$.

В. Пусть $\operatorname{dim} X<2 \min \{k, l\}$. Тогда следующие условия эквивалентны:

(i) $A_{k}$ - основа некоторого ПРА над $X$;

(ii) для произвольного $m$, удовлетворяющего условию $2 m>\operatorname{dim} X$, существует расслоение на $m$-алгебры $B_{m}$ такое, что $A_{k} \otimes \widetilde{M}_{m} \cong B_{m} \otimes \widetilde{M}_{k}$;

(iii) для некоторого $m$ такого, что $(k, m)=1: A_{k} \otimes \widetilde{M}_{m} \cong B_{m} \otimes \widetilde{M}_{k}$.

Более того, по паре расслоений $A_{k}$ и $B_{m}$ над $X$ такой, что $(k, m)=1$ и $A_{k} \otimes \widetilde{M}_{m} \cong B_{m} \otimes \widetilde{M}_{k}$, однозначно восстанавливается класс стабильной эквивалентности ПРА над $X$, который имеет (для произвольного достаточно большого $n$ такого, что $(k m, n)=1$ ) в качестве представителей ПРА $\left(A_{k}, \mu, \widetilde{M}_{k n}\right)$ и $\left(B_{m}, \nu, \widetilde{M}_{m n}\right)$ (для некоторых $\mu$ и $\left.\nu\right)$.

Работа выполнена при частичной поддержке Российского фонда фундаментальных исследований (грант № 99-01-01202). 
С. На множестве классов стабильной эквивалентности ПРА над $X$ с помощью операции тензорного произведения расслоений можно ввести структуру абелевой группы $A B^{1}(X)$. Соответствующее классифицирующее $H$-пространство $\lim _{(k, l)=1} G r_{k, k l}$ имеет такие же гомотопичес-
кие группы, как и $B S U$.

Используя эти результаты, построим биекцию между множеством классов обычной стабильной эквивалентности $S U$-расслоений $\widehat{K S U}(X)$ и множеством классов эквивалентности ПРА из определения $2 A B^{1}(X)$ и, тем самым, докажем гомотопическую эквивалентность $\lim _{(k, l)=1} G r_{k, k l}$ и $B S U$ (см. пункт C).

Пусть $\xi_{k}-k$-мерное комплексное $S U(k)$-расслоение над $X, \operatorname{dim} X<2 k$. Рассмотрим пару

$$
\xi_{k} \otimes[k]-[k(k-1)], \quad\left(\xi_{k} \oplus[m-k]\right) \otimes[m]-[m(m-1)]
$$

(здесь $[n]$ - тривиальное расслоение ранга $n$ над $X$ ) виртуальных расслоений виртуальной размерности $k$ и $m$ соответственно, где $(k, m)=1$ и $m>k$. Обозначим через $\xi_{k}^{\text {ad }},\left(\xi_{k} \oplus[m-k]\right)$ ad геометрических представителей этих классов размерностей $k$ и $m$ соответственно, которые существуют в силу условия на размерность $X([2])$.

ПрЕДЛОЖЕниЕ $3 .\left(\right.$ End $\left.\xi_{k}^{\text {ad }}\right) \otimes \widetilde{M}_{m} \cong\left(\operatorname{End}\left(\xi_{k} \oplus[m-k]\right)^{\mathrm{ad}}\right) \otimes \widetilde{M}_{k}$.

ДокАЗАТЕЛЬСТво. Прямым вычислением проверяется, что имеет место следующий изоморфизм: $\chi \otimes[m] \cong\left(\xi_{k} \oplus[m-k]\right)^{\mathrm{ad}} \otimes[k]$.

Согласно пункту В и доказанному предложению, по паре $\chi,\left(\xi_{k} \oplus[m-k]\right)^{\text {ad }}$ однозначно восстанавливается некоторый класс стабильной эквивалентности ПРА над $X$. Легко видеть, что он не зависит от выбора представителя класса $\widehat{K S U}$-эквивалентности расслоения $\xi_{k}$. Следовательно, имеет место

ПРЕДЛОЖЕНИЕ 4. Корректно определено отображение множеств

$$
\phi: \widetilde{K S U}(X) \rightarrow A B^{1}(X), \quad \xi_{k} \mapsto\left(A_{k}, B_{m}\right),
$$

əдe $A_{k}=\operatorname{End} \chi, B_{m}=\operatorname{End}\left(\xi_{k} \oplus[m-k]\right)^{\mathrm{ad}},(k, m)=1, \operatorname{dim} X<2 \min \{k, m\}$.

Пусть $\left(\xi_{k}, \xi_{m}\right)$ - пара $S U(k)$ - и $S U(m)$-расслоений соответственно $((k, m)=1)$, причем $\xi_{k} \otimes[m] \cong \xi_{m} \otimes[k]$. Возьмем $l$ и $n$ такие, что $k l+m n=1$. Тогда в группе $K S U(X)$ выполняется равенство $\xi_{k}=k l \xi_{k}+m n \xi_{k}=k l \xi_{k}+k n \xi_{m}=k\left(l \xi_{k}+n \xi_{m}\right)$ и аналогично $\xi_{m}=m\left(l \xi_{k}+n \xi_{m}\right)$ (здесь мы классы стабильной эквивалентности расслоений обозначаем теми же символами, что и их представителей), т.е. $\xi_{k}=k \eta, \xi_{m}=m \eta$ для одномерного виртуального $S U$-расслоения $\eta$ $\left(\eta=l \xi_{k}+n \xi_{m}\right)$. Очевидно, что сопоставление $\left(A_{k}, B_{m}\right) \mapsto \eta\left(\right.$ где $A_{k}=$ End $\xi_{k}, B_{m}=$ End $\left.\xi_{m}\right)$ определяет искомое отображение $\psi$, обратное к $\phi$. Тем самым получаем

ПРЕДЛОжение 5. Пространства $\lim _{(k, l)=1} G r_{k, k l}$ (см. пункт C) u BSU гомотопически әквивалентны.

Обозначим через $B S U_{\otimes}$ пространство $B S U$ со структурой $H$-пространства, индуцированной тензорным произведением виртуальных $S U$-расслоений виртуальной размерности один [3].

Теорема 6. H-пространство $\lim _{(k, l)=1} G r_{k, k l}$ с операцией, индуцированной тензор-

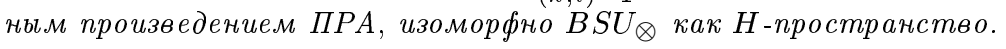

Автор выражает глубокую благодарность В. М. Мануйлову, А. С. Мищенко и Е. В. Троицкому за полезные обсуждения.

\section{СПИСОК ЛИТЕРАТУРЫ}

[1] Ершов А. В. // Вестник МГУ. Сер. 1. Матем., мех. 1999. №6. С. 56-60. [2] Хьюзмоллер Д. Расслоенные пространства. М.: Мир, 1970. [3] А дамс Дж. Ф. Бесконечнократные пространства петель. М.: Мир, 1982. 